\title{
Patterns of olfactory dysfunctions in patients with Parkinson disease
}

\author{
Khaled Hussein Rashed ${ }^{1}$, Wafik Said Bahnasy ${ }^{1 *}$ (D), Yasser Abo Elfotoh El-Heneedy ${ }^{1}$, Ehab Ahmed Shawky El-Seidy ${ }^{1}$ \\ Mohamed Osama Tomoum², Mohamed Adel Eltomey ${ }^{3}$ and Shereen Ahmed ELAhwal ${ }^{1}$
}

\begin{abstract}
Background: Olfactory dysfunction (OD) is a well-established nonmotor manifestations (NMM) of Parkinson disease (PD) which needs objective assessment for better understanding of the disease pathogenesis. The aim of this work was quantitative and qualitative assessment of olfactory performance in newly diagnosed PD patients.

Methods: This study was performed on 32 recently diagnosed PD patients and 24 healthy controls subjects (HCS) submitted to unified Parkinson's disease rating scale-III (UPDRS-III), extended n-butanol Sniffin' Sticks test (SST) and olfactory bulbs volumetry (OBV).

Results: There were significant decreases in SST threshold, discrimination, identification, and TDI variables as well as OBV in PD patients compared to HCS. The olfactory performance was negatively correlated with disease duration but had no relation with PD severity as well as motor subtype.

Conclusion: $\mathrm{OD}$ is highly prevalent during the early stages of PD which is both measurable and specific with identification and discrimination impairments to certain odors which makes smell performance testing an important step in PD patients' evaluation.
\end{abstract}

Keywords: Parkinson disease, Non-motor manifestations, Olfactory dysfunction, Sniffin' Sticks test, Olfactory bulb volumetry

\section{Introduction}

Parkinson disease (PD) is the 2nd most prevalent neurodegenerative disorder of elderly affecting $1-2 \%$ of the people $>65$ years and $0.3 \%$ of the general population [1]. The disease is now well known by its non-motor manifestations (NMM) which often start very early in the prodromal premotor phase while their absence is now considered as a red flag of PD diagnosis [2]. The NMM represents extra-nigral $\alpha$-synucleinopathies which have their own routes and rates of progression. PD could be divided to several NMM variants: brainstem dominant phenotype (Park. sleep and Park. autonomic variants), limbic dominant phenotype (Park. fatigue, Park. pain/ allodynia, and Park. depression/anxiety variants), and

\footnotetext{
* Correspondence: wafiq.elbahnasi@med.tanta.edu.eg

'Department of Neurology, Faculty of Medicine, Tanta University, Tanta 31527, Egypt

Full list of author information is available at the end of the article
}

cortical dominant phenotype (Park. cognition/fall and Park. apathy variants) [3].

Olfactory dysfunction (OD) due to PD is defined as unequivocal hyposmia, after adjustment for age. The classic Braak staging of PD included OD within the brainstem dominant phenotype, but it is highly frequent that could be found in other NMM variants. He supposed that Lewy bodies deposition starts in the olfactory bulb and anterior olfactory nucleus then progresses to the limbic system several years before substantia nigra (SN) involvement [4]. OD is now considered as a supportive criterion for PD diagnosis due to its high sensitivity and specificity $(>80 \%$ ) [5]. Olfactory performance in PD patients is often studied in holistic all or none method with little attention to the peculiarities as well as the prognostic value of OD. This subjective assessment is often inaccurate with limited capabilities in 
characterization of the specific nature of such neurodegenerative disorder [6].

\begin{abstract}
Aim of the work
Aim of the work was to assess the olfactory dysfunctions in recently diagnosed PD patients in quantitative and qualitative manner as well as their correlation with the disease severity, duration, and the dominating motor variant.
\end{abstract}

\section{Methods}

The study was an observational cross-sectional casecontrol study conducted on 32 consecutives recently diagnosed PD patients (within 1-year of motor manifestations onset) recruited from the movement disorder clinic, Neuropsychiatry Department, Tanta University Hospitals in the period from 1 October 2017 to the end of January 2019. The inclusion criteria encompassed PD patients within 3-years of their MM onset and aged 50-65 years (to avoid the age-related effect on olfactory functions). PD was diagnosed according to the International Parkinson and Movement Disorder Society (MDS) clinical diagnostic criteria for PD, 2015 [7]. Twenty-four age, sex, smoking index, and residence matched healthy control subjects (HCS) lacked any family history of neurodegenerative disorders were also included.

Exclusion criteria comprised PD patients with motor complications, secondary or heredodegenerative parkinsonism, and non-cooperative patients as well as those with comorbid medical/neurological illness, MRI contraindication, or sino-nasal disorders affecting smell. The study protocol was approved by the Research Ethics Committee and Quality Assurance Unit, Faculty of Medicine, Tanta University, participation was voluntary, and informed consents were obtained from all participants before engagement in the study.

Included patients were told to stop PD therapy (Ldopa, dopaminergic, and anticholinergic drugs), after which the unified Parkinson's disease rating scale-III (UPDRS-III) was done to assess disease severity as well as determination of the motor variants by the estimating the ratio of the 11 tremor score items sum by the 5 postural instability gait difficulty (PIGD) sum [8]. Patients had ratios $\geq 1.15$ were considered tremor dominant (TD) variant while patients with ratios $\leq 0.9$ were considered PIGD variants. Patients had ratios $>0.9$ and $<$ 1.15 were identified as mixed intermediate variants [9]. The studied patients' sample included $22 \mathrm{TD}$ (group Ia) and 10 PIGD (group Ib) dominant PD variants.

Olfactory performance was assessed using extended nbutanol, Burghart Sniffin' Sticks test (SST), LA-13-00134, Wedel, Germany, by mean of the 3 smell subtests; threshold, discrimination, and identification regarding to Rumeau and colleagues, 2016 [10]. The sum of the previous 3-scores; TDI (threshold, discrimination, and identification) could quantify the olfactory performance as follow: $\geq 31$ normosmia, 16-30 hyposmia, and $\leq 15$ functional anosmia [10] (Additional file 1).

Brain MRI was performed by $1.5 \mathrm{~T}$ Toshiba Titan Vantage MRI machine (Toshiba Medical Systems, Japan) using the Atlas head coil. Imaging protocol included 3D coronal T2-weighted steady state free precession (SSFP) sequence TR 10/TE 5, slice thickness $0.5 \mathrm{~mm}$, and slice spacing $0.5 \mathrm{~mm}$. Olfactory bulb volumes (OBV) were measured using the 3D slicer software for segmentation of the olfactory bulb and volume calculation using manual tracing of the right and left bulbs in cuts (Fig. 1).

For each parameter of SST as well as the OBV, every subject was represented by the mean value of each bilaterally assessed parameters. Olfactory performances in PD patients were considered asymmetrical if the side to side difference exceeds 3-points in TDI and/or $3.5 \mathrm{~mm}^{3}$ for OBV (1.5 times the standard deviation of normal).

Statistical analysis was conducted using SPSS Prism, version 20, 2013, created by IBM, Illinois, Chicago, USA. Chi-square was used for categorical variables statistical differences and student $t$ test for numerical ones. Correlation analysis was performed using Pearson's correlation test. $P$ value $<0.05$ was considered statistically significant.

\section{Results}

The studied PD patients' mean \pm SD of the age was 57.7 \pm 3.5 years, 19 males (59.4\%), 13 females (40.6\%); the disease duration was $2.1 \pm 0.6$ years and the mean UPDRS-III was $48.4 \pm 20.9 ; 22$ (68.75\%) were TD- and 10 (31.25\%) were PIGD-PD variants.

The results showed that only $10(31.25 \%)$ of patients were aware of their OD while the remaining were not certain about their smelling problem. Quantitative olfactory performance measured by the SST total score (TDI) showed that none of the PD patients had normal olfactory performance (TDI $\geq 31), 13$ (40.6\%) were hyposmic, and $19(59.4 \%)$ had functional anosmia.

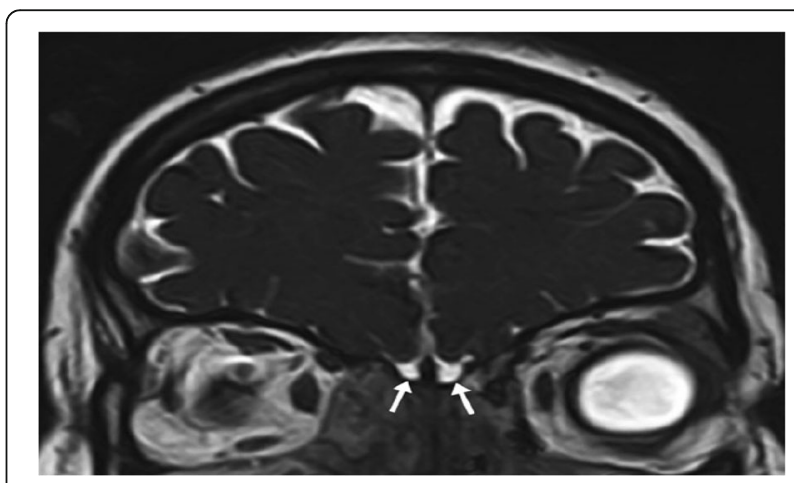

Fig. 1 Coronal T2-WI showing segmentation of the olfactory bulb for volumetric assessment 
Table 1 Statistical analysis of olfactory performance among Parkinson disease patients (group I) and healthy control subjects (group II)

\begin{tabular}{|c|c|c|c|c|}
\hline & \multirow{2}{*}{$\begin{array}{l}\text { Group I ( } \boldsymbol{n} \text {, } \\
32)\end{array}$} & \multirow{2}{*}{$\begin{array}{l}\text { Group II ( } \boldsymbol{n}, \\
24)\end{array}$} & \multicolumn{2}{|l|}{$\boldsymbol{t}$ test } \\
\hline & & & $\boldsymbol{t}$ value & $\boldsymbol{p}$ value \\
\hline Threshold & $3.72 \pm 1.40$ & $9.38 \pm 1.71$ & 13.6 & $<0.001^{*}$ \\
\hline Identification & $6.53 \pm 2.02$ & $6.53 \pm 2.02$ & 11.878 & $<0.001^{*}$ \\
\hline Discrimination & $5.97 \pm 2.06$ & $11.29 \pm 1.33$ & 11.047 & $<0.001 *$ \\
\hline TDI total score & $16.22 \pm 5.34$ & $32.75 \pm 3.72$ & 12.976 & $<0.001 *$ \\
\hline OBV $\left(\mathrm{mm}^{3}\right)$ & $32.25 \pm 4.57$ & $50.35 \pm 3.12$ & 16.702 & $<0.001^{*}$ \\
\hline
\end{tabular}

$O B V$ olfactory bulb volumetry, $T D I$ threshold, discrimination, identification *Significant

The study showed a significant decrease in each of SST threshold, discrimination, identification, and TDI in PD patients compared to HCS with $p$ values $\leq 0.001$. At the same time, the threshold was the most affected subtest $(t$ value $=13.6)$ compared to identification and discrimination subtests $(t$ value $=11.878$ and 11.047 respectively). Regarding OBV, there was a significant decrease in PD patients compared to HCS with $p$ value $\leq$ 0.001 (Table 1 and Fig. 2).
The results also showed that 5 (15.6\%) PD patients had asymmetrical OD with smell deficit was higher ipsilateral to the more affected side of motor manifestations. This asymmetrical affection could be identified in patients with mild hyposmia and very short disease duration while patients with functional anosmia revealed nearly symmetrically olfactory impairment. At the same time, SST parameters (threshold, identification, discrimination, and TDI), and OBV showed nonsignificant differences between TD and PIGD variants of PD with $p$ value $>0.05$ (Table 2 ).

Regarding the Sniffin' Sticks identification subtest, there were highly significant decreases in items $2,3,7$, 14,15 , and 16 correct responses (leather, cinnamon, licorice, rose, aniseed, and fish) by PD patients compared to HCS with $p$ values $<0.001$. At the same time, items 1 , 5,10 , and 13 (orange, banana, coffee, and pineapple) identification revealed mildly significant impairment in PD patients compared to HCS with $p$ values: 0.018 , $0.013,0.037$, and 0.01 respectively. On the other hand, PD patients showed non-significant differences in items $4,6,8,9,11$, and 12 identification (peppermint, lemon,

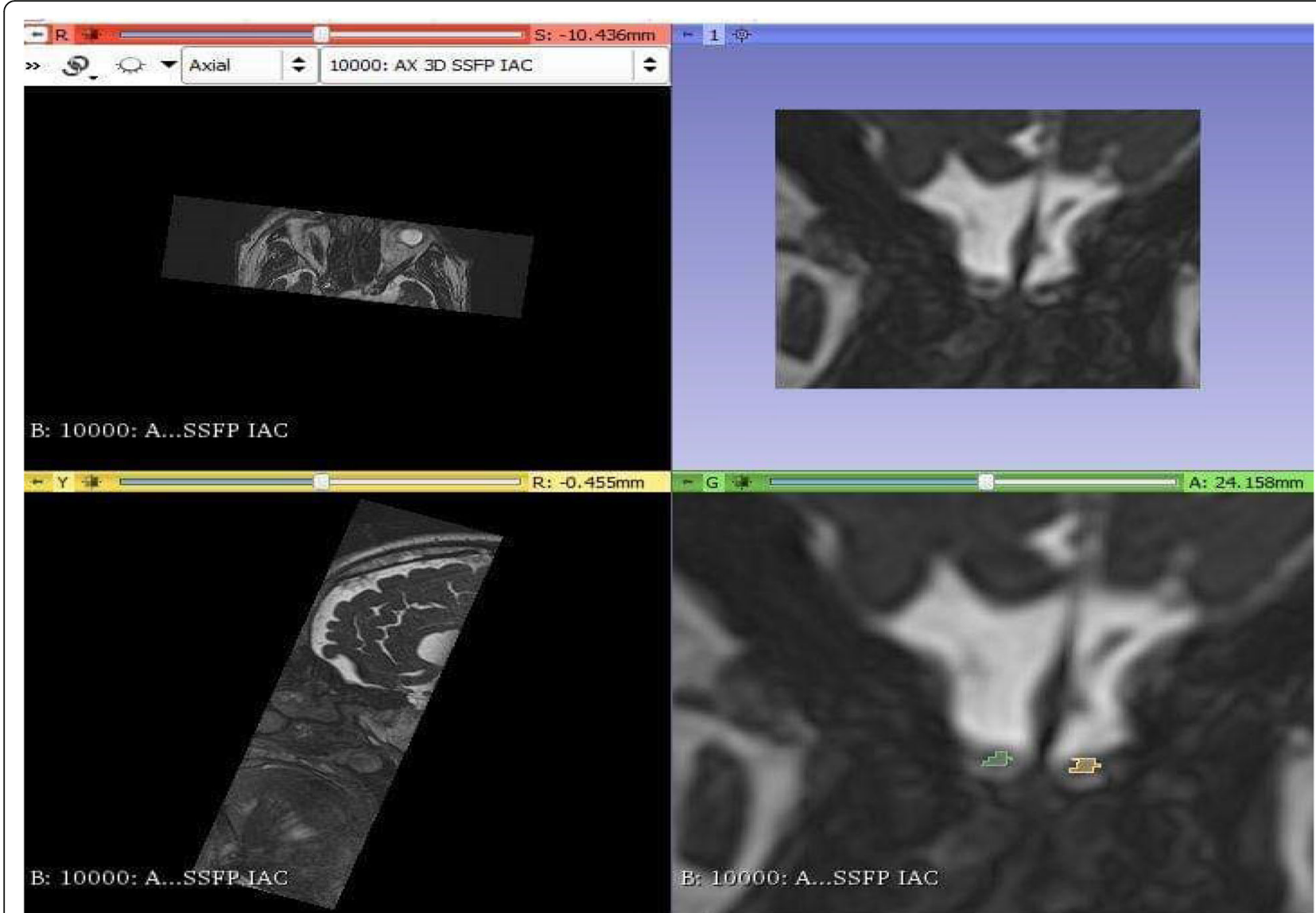

Fig. 2 Three-dimension T2-weighted steady state free precession olfactory bulb volumetry showing right and left olfactory bulbs volumes of 27.1 and $29.8 \mathrm{~mm}^{3}$ respectively 
Table 2 Statistical analysis of Sniffin Stick test and olfactory bulb volumetry among tremor dominant (group la) and postural instability gait difficulty (group Ib) variants of Parkinson disease patients

\begin{tabular}{|c|c|c|c|c|}
\hline & \multirow{2}{*}{$\begin{array}{l}\text { Group la (n, } \\
22)\end{array}$} & \multirow{2}{*}{$\begin{array}{l}\text { Group Ib (n, } \\
10)\end{array}$} & \multicolumn{2}{|l|}{$\boldsymbol{t}$ test } \\
\hline & & & $\boldsymbol{t}$ value & $\boldsymbol{p}$ value \\
\hline Threshold & $3.77 \pm 1.27$ & $3.60 \pm 1.71$ & 0.102 & 0.752 \\
\hline Identification & $6.64 \pm 1.87$ & $6.30 \pm 2.41$ & 0.186 & 0.669 \\
\hline Discrimination & $6.00 \pm 1.90$ & $5.90 \pm 2.47$ & 0.016 & 0.901 \\
\hline TDI total score & $16.41 \pm 4.90$ & $15.80 \pm 6.48$ & 0.087 & 0.770 \\
\hline OBV $\left(\mathrm{mm}^{3}\right)$ & $31.84 \pm 4.43$ & $33.14 \pm 4.98$ & 0.548 & 0.465 \\
\hline
\end{tabular}

$O B V$ olfactory bulb volumetry, $T D I$ threshold, discrimination, identification

turpentine, garlic, apple, and clove odors) compared to with $p$ values $>0.05$ (Table 3 ).

The results of the present study also showed significant negative correlations between disease duration and SST parameters (threshold, identification, discrimination, and TDI) as well as OBV with $p$ value $<0.001$ for each. On the other hand, the UPDRS-III showed a non-significant correlation with the same variables (Table 4, Fig. 3).

\section{Discussion}

Olfactory dysfunction had described as one of the most important and pathognomonic NMM of PD. The present work showed that all included PD patients

Table 3 Percent of correct responses in the identification Sniffin' Sticks subtest among Parkinson disease patients (group I) and healthy control subjects (group II)

\begin{tabular}{llllll}
\hline & Target odor & Group I $(n$, & Group II $(n$, & \multicolumn{2}{l}{ Chi square } \\
\cline { 5 - 7 } & & $32)$ & $24)$ & $X^{2}$ & $p$ value \\
\hline Item-1 & Orange & $17(53.1 \%)$ & $20(83.3 \%)$ & 5.579 & $0.018^{*}$ \\
Item-2 & Leather & $13(40.6 \%)$ & $21(87.5 \%)$ & 12.629 & $<0.001^{*}$ \\
Item-3 & Cinnamon & $1(3.1 \%)$ & $17(70.8 \%)$ & 28.832 & $<0.001^{*}$ \\
Item-4 & Peppermint & $26(81.25 \%)$ & $23(95.8 \%)$ & 2.668 & 0.102 \\
Item-5 & Banana & $20(62.5 \%)$ & $22(91.7 \%)$ & 6.219 & $0.013^{*}$ \\
Item-6 & Lemon & $14(43.75 \%)$ & $15(62.5 \%)$ & 1.927 & 0.165 \\
Item-7 & Licorice & $11(34.3 \%)$ & $19(79.2 \%)$ & 11.061 & $<0.001^{*}$ \\
Item-8 & Turpentine & $17(53.1 \%)$ & $14(58.3 \%)$ & 0.152 & 0.698 \\
Item-9 & Garlic & $21(65.6 \%)$ & $20(83.3 \%)$ & 2.189 & 0.139 \\
Item-10 & Coffee & $20(62.5 \%)$ & $21(87.5 \%)$ & 4.371 & $0.037^{*}$ \\
Item-11 & Apple & $15(46.9 \%)$ & $14(58.3 \%)$ & 0.719 & 0.396 \\
Item-12 & Clove & $23(71.9 \%)$ & $22(91.7 \%)$ & 3.401 & 0.065 \\
Item-13 & Pineapple & $13(40.6 \%)$ & $18(75 \%)$ & 6.559 & $0.010^{*}$ \\
Item-14 & Rose & $10(31.25 \%)$ & $20(83.3 \%)$ & 14.962 & $<0.001^{*}$ \\
Item-15 & Aniseed & $3(9.4 \%)$ & $21(87.5 \%)$ & 34.179 & $<0.001^{*}$ \\
Item-16 & Fish & $14(43.75 \%)$ & $23(95.8 \%)$ & 16.601 & $<0.001^{*}$ \\
\hline *Significant & & & &
\end{tabular}

*Significant suffered of severe quantitative olfactory impairment with their TDI score located within the categories of hyposmia or functional anosmia. These results are in agreement with that of Fullard and colleagues [11] as well as Oh and colleagues [12] who stated that smell dysfunction is consistent NMM of PD affecting > $90 \%$ of cases even in their early disease stages.

The results of the present work also showed profound smell dysfunction in PD patients with very short disease duration $(<1$ year) and it was positively correlated with the disease duration pointing to the premotor onset of OD as well as its faster rate of progression than the MM. These findings are in accordance with that of Tremblay and colleagues [13] as well as Wen and colleagues [14] who stated that OD is a consistent NMM of PD which is evident in de novo cases as well as during the premotor phase. At the same time, abnormal network connectivity (small worldness and clustering coefficient) among areas involved in olfaction could be determined very early during the prodromal phase of PD.

The study showed that a big sector of PD patients lack self-reported olfactory dysfunction and was much preoccupied by the motor symptoms and supposed that the olfactory problem is not part of PD manifestations. This result is passing with the work of Seppi and colleagues [15] who stated that despite the marked in smelling performance, most PD patients are not mindful about their OD problem except with objective testing and they become only symptomatic with the beginning of MM. This observation could be explained by the lack of awareness, the non-neurological consultations, and the limited tools of its management. On the other hand, García and colleagues [16] revealed that most PD patients are aware of their anosmia which is highly troublesome and impairs their quality of life. This discrepancy is most probably related to different cultural knowledge and possibly due to their dealing with the NMM in a collective manner used the non-motor symptom total score.

The results showed that few included patients, with short disease duration and mild hyposmia, had asymmetrical olfactory performance with more deficit ipsilateral to the more affected motoric side. This notice points to the expansion of PD asymmetry to the nonmotor manifestations including smelling, but this asymmetrical OD tends to be short lived and quickly gets symmetrical with the progression of the disease. These results are in accordance with the work of Hobson and colleagues [17] as well as Riederer and colleagues [18] who stated that asymmetry is a distinctive feature of PD disease which does not only involve the MM but also extend to the non-motor ones including OD. They also demonstrated that olfactory performance is usually more affected ipsilateral to the 
Table 4 Correlations between each of Sniffin Stick test and olfactory bulb volumetry with disease duration and severity among Parkinson disease patients' group

\begin{tabular}{llllll}
\hline & \multicolumn{2}{l}{ Duration } & & \multicolumn{2}{l}{ UPDRS-III } \\
\cline { 2 - 3 } & $r$ & $p$ & & $r$ & $p$ \\
\hline Threshold & -0.729 & $<0.001^{*}$ & & -0.003 & 0.988 \\
Identification & -0.711 & $<0.001^{*}$ & & -0.086 & 0.638 \\
Discrimination & -0.765 & $<0.001^{*}$ & & -0.043 & 0.814 \\
TDI total score & -0.754 & $<0.001^{*}$ & -0.050 & 0.786 \\
OBV $\left(\mathrm{mm}^{3}\right.$ ) & -0.798 & $<0.001^{*}$ & -0.120 & 0.514 \\
\hline UPDRD-III unified Parkinson's disease rating scale-III & & \\
*Significant & & & &
\end{tabular}

more affected limb and contralateral to the SN pars compacta neuronal loss as well as putamenal hypofunction evidenced by high field strength MRI (swallow tail sign) as well as functional DAT - SPECT and 18 F-DOPA - PET assessments.

The lack of a significant correlation between SST variables with disease severity (UPDRS-III) as well as the non-significant difference between TD and PIGD motor variants regarding their smelling performance signifies that OD has an extranigral route of progression. These data are in harmony with the studies of Halliday and colleagues [19] as well as Gunzler and colleagues [20] who declared that PD is a multiorgan and multi-peptide related disorder with the non-dopaminergic extranigral involvement sponsored for its NMM including OD.

The study showed that PD had selective qualitative hyposmia to certain odors in the identification subtest (leather, cinnamon, licorice, rose, aniseed, and fish) pointing to the possible vulnerability of certain olfactory bulb receptor neurons to PD-related synucleinopathies. These results are in accordance with that of Boesveldt and colleagues [21] as well as Leonhardt and colleagues [22] who stated that hyposmia to specific odors could be noticed in PD including pizza and wintergreen in daily life as well as aniseed, cinnamon, and licorice in the SST. They also presumed that such olfactory identification problems could be used as a biomarker distinguishing PD from other neurodegenerative disorders. On the other hand, Mantri and colleagues [23] supposed that the different performance of PD patients in the identification of certain odors could be related to cultural disparities rather than specific predilection of $\alpha$ synucleinopathy to specific olfactory receptors. However, the results of this study contravene with the later possibility because the identification test was performed on PD and HCS of the same culture while the SST rater was blind regarding the results of other studies which are matching with this study in weak aniseed, cinnamon, and licorice odor identification by PD patients who could identify peppermint, lemon, turpentine, garlic, and clove odors more easily.

The results of the present work showed reduced OBV in PD patients which is sometimes asymmetrical especially in PD cases with hyposmia. These data are in agreement with that of Sengoku and colleagues [24] as well as Park and colleagues [25] who declared that olfactory bulb morphological abnormalities could be distinguished during the early prodromal premotor phase of PD. At the same time, Hwang and colleagues [26] identified that the presence of asymmetry in the primary olfactory structures including the olfactory bulb or its input to the primary olfactory cortex or the medio-dorsal thalamic nucleus could be a valuable biomarker differentiating PD from other PD-related neurodegenerative disorders

\section{Conclusion}

Olfactory impairment as well as OBV are valuable diagnostic biomarker of PD. Olfactory performance in PD patients had its own specificities including tendency to
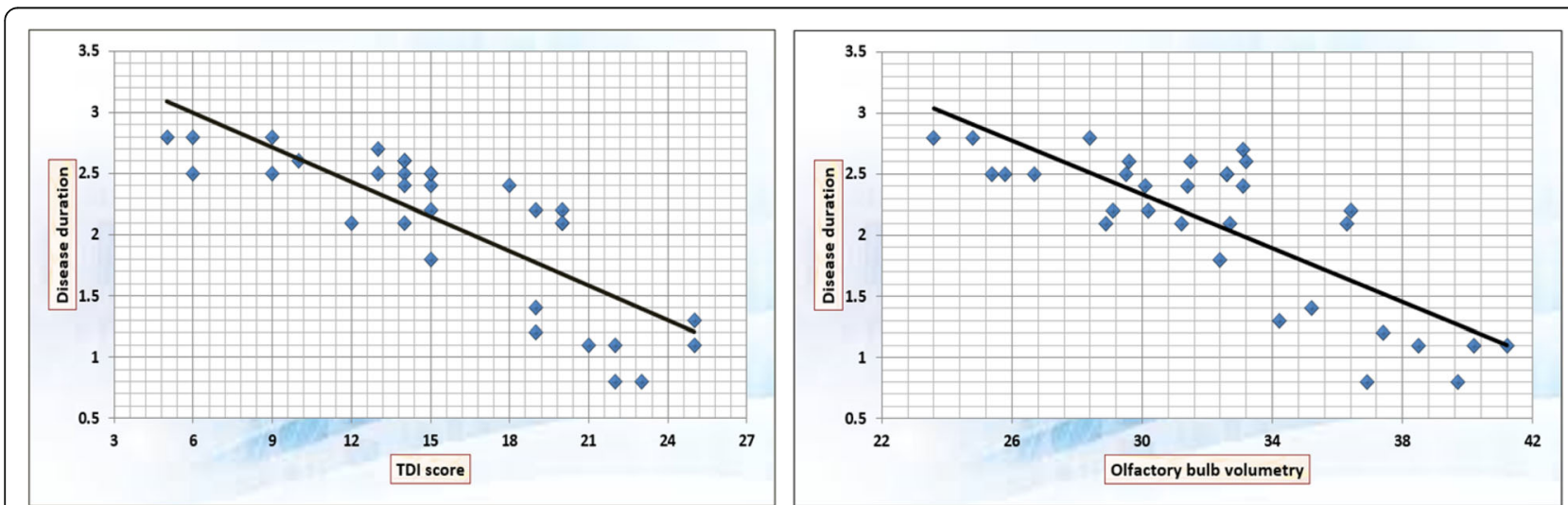

Fig. 3 Negative correlation between Parkinson's disease duration and each of threshold, discrimination, identification global score (left), and the olfactory bulb volumetry (right) 
asymmetrical affection and more involvement of leather, cinnamon, licorice, rose, aniseed, and fish odors.

\section{Recommendations}

Validated, inexpensive, and easily applicable olfactory tests are needed as a screening tool for identifying prodromal, pre-motor PD cases to prepare the floor for the introduction of disease-modifying therapy. At the same time, further studying of the mode of $\alpha$-synucleinopathy spread among the olfactory bulb and tracts may improve our understanding of the physiology of olfaction as well as the pathogenesis of PD including the miracle of asymmetry.

\section{Limitations}

Higher field strength MRI machine with better OBV assessment using 3-D automated techniques as well as diffusion tensor imaging could provide more details about the characters olfactory bulb degeneration in PD. Longitudinal study of patients is further needed to determine the value of early OD as a prognostic biomarker of PD as well as diagnosis of HCS who may be in a prodromal stage of PD or any other neurodegenerative disorder affecting smell. This issue will be covered in the 2nd phase of this work.

\section{Supplementary information}

Supplementary information accompanies this paper at https://doi.org/10. 1186/s41983-020-00205-z.

Additional file 1. Sniffin' Sticks test.

\section{Abbreviations \\ HCS: Healthy control subjects; MDS: Movement disorder society; MM: Motor manifestations; NMM: Nonmotor manifestations; OBV: Olfactory bulb volumetry; OD: Olfactory dysfunction; PD: Parkinson disease; PIGD: Postural instability gait dominant; SN: Substantia nigra; SST: Sniffin' Sticks test; TDI: Threshold, discrimination, and identification; TD: Tremor dominant; UPDRS-III: Unified Parkinson's disease rating scale-III}

\section{Acknowledgements}

We would like to thank the neurology and otolaryngology medical and paramedical staff, Tanta University Hospitals for their great help in patients' selection and evaluation.

\section{Consent of publication}

Not applicable.

\section{Authors' contributions}

KHR participated in the study's design, patients' selection, statistical analysis, data analysis, references collection, manuscript writing and revision, and final approval. WSB participated in the study's idea, design, patients' selection, neurological examination, statistical analysis, data analysis, references collection, manuscript writing, revision, and final approval. YAE participated in the study's idea and design, patients' assessment and inclusion, data analysis, statistical analysis, references collection, manuscript writing, revision, and final approval. EASE participated in the study's idea, design, patients' selection, and evaluation, data analysis, references collection, manuscript revision, and final approval. MOT participated in the study's idea and design, patients' assessment and inclusion, Sniffin' Sticks test performance, data analysis, references collection, manuscript writing, revision, and final approval. MAE participated in the study's design, patients' assessment, olfactory bulb volumetry performance, manuscript revision, and final approval. SAE participated in the study's design, patients' selection, statistical analysis, data analysis, references collection, manuscript writing and revision, and final approval.

\section{Funding}

No funding had been received.

\section{Availability of data and materials}

The datasets used and/or analyzed during the current study are available from the corresponding author on reasonable request.

\section{Ethics approval and consent to participate}

The manuscript was approved from The Research Ethics Committee and Quality Assurance Unit, Faculty of Medicine, Tanta University.

The URL: http://tqac.tanta.edu.eg/new-tqac/

QualityAssuranceUnit@hotmail.com

Approval Code: 31277/12/16

Name of the PI: Yasser Abo Elfotoh El-Heneedy.

Name of the department: Neuropsychiatry.

Type of the research: Promotion research.

Date of approval: November 2016.

The study's protocol had approved by The Research Ethics Committee and Quality Assurance Unit, Faculty of Medicine, Tanta University. Participation was voluntary; informed consents were approved by all participants and any possible risks were clarified.

\section{Competing interests}

All authors disclose that they have no competing interests related to the study.

\section{Author details}

'Department of Neurology, Faculty of Medicine, Tanta University, Tanta 31527, Egypt. ${ }^{2}$ Otolaryngology/Head and Neck Surgery Department, Faculty of Medicine, Tanta University, Tanta 31527, Egypt. ${ }^{3}$ Department of Diagnostic Radiology, Faculty of Medicine, Tanta University, Tanta, Egypt.

Received: 25 March 2020 Accepted: 25 June 2020

Published online: 10 July 2020

\section{References}

1. Di Battista ME, Cova I, Rubino A, Papi CP, Alampi G, Purcaro C, et al. Intercepting Parkinson disease non-motor subtypes: a proof-of-principle study in a clinical setting. J Neurol Sci. 2018;388:186-91. https://doi.org/10. 1016/j.jns.2018.03.024.

2. Ragab OA, Elheneedy YA, Bahnasy WS, Ragab et al. Non-motor symptoms in newly diagnosed Parkinson's disease patients. Egyptian J Neurol Psychiatry Neurosurg. 2019; 55:24. doi: 10.1186/s41983-019-0070-2.

3. Sauerbier A, Jenner P, Todorova A, Chaudhuri KR. Non motor subtypes and Parkinson's disease. Parkinsonism Relat Disord. 2016;22:S41-6. https://doi. org/10.1016/j.parkreldis.2015.09.027.

4. Mantri S, Morley JF, Siderowf AD. The importance of preclinical diagnostics in Parkinson disease. Parkinsonism Relat Disord. 2018; doi: 10.1016/j. parkreldis.2018.09.011.

5. Versace V, Langthaler PB, Sebastianelli L, Höller Y, Brigo F, Orioli A, et al. Impaired cholinergic transmission in patients with Parkinson's disease and olfactory dysfunction. J Neurol Sci. 2017;377:55-61. https://doi.org/10.1016/j. jns.2017.03.049.

6. Reich SG, Savitt JM. Parkinson's disease. Med Clin N Am. 2019;103(2):337-50. https://doi.org/10.1016/..mcna.2018.10.014.

7. Postuma RB, Berg D, Stern M, Poewe W, Olanow CW, Oertel W, et al. MDS clinical diagnostic criteria for Parkinson's disease. Mov Disord. 2015;30(12): 1591-9. https://doi.org/10.1002/mds.26424.

8. Goetz CG, Tilley BC, Shaftman SR, Stebbins GT, Fahn S, Martinez-Martin P, et al. Movement disorder society-sponsored revision of the unified Parkinson's disease rating scale (MDS-UPDRS): process, format, and clinometric testing plan. Mov Disord. 2008;23(15):2129-70. https://doi.org/ $10.1002 / \mathrm{mds} .22340$.

9. Choi SM, Kim BC, Cho BH, Kang KW, Choi KH, Kim JT, et al. Comparison of two motor subtype classifications in de novo Parkinson's disease. 
Parkinsonism Relat Disord. 2018;54:74-8. https://doi.org/10.1016/j.parkreldis. 2018.04.021.

10. Rumeau C, Nguyen DT, Jankowski R. How to assess olfactory performance with the Sniffin' Sticks test. Eur Ann Otorhinolaryngol Head Neck Dis. 2016; 133:203-6. https://doi.org/10.1016/j.anorl.2015.08.004.

11. Fullard ME, Morley JF, Duda JE. Olfactory dysfunction as an early biomarker in Parkinson's disease. Neurosci Bull. 2017;33(5):515-25. https://doi.org/10. 1007/s12264-017-0170-x.

12. Oh YS, Kim JS, Hwang EJ, Lyooc CH. Striatal dopamine uptake and olfactory dysfunction in patients with early Parkinson's disease. Parkinsonism Relat Disord. 2018;56:47-51. https://doi.org/10.1016/j.parkreldis.2018.06.022.

13. Tremblay G, Martel PD, Frasnelli J. Trigeminal system in Parkinson's disease: a potential avenue to detect Parkinson-specific olfactory dysfunction. Parkinsonism Relat Disord. 2017;44:85-90. https://doi.org/10.1016/j.parkreldis. 2017.09.010

14. Wen MC, Heng HSE, Hsu JL, Xu Z, Liew GM, Au WL, et al. Structural connectome alterations in prodromal and de novo Parkinson's disease patients. Parkinsonism Relat Disord. 2017;45:21-7. https://doi.org/10.1016/j. parkreldis.2017.09.019.

15. Seppi K, Chaudhuri KR, Coelho M, Fox SH, Regina Katzenschlager R, Lloret $\mathrm{SP}$, et al. Update on treatments for nonmotor symptoms of Parkinson's disease: an evidence-based medicine review. Mov Disord. 2019;34(2):180-98. https://doi.org/10.1002/mds.27602.

16. García DS, Jesús S, Aguilar M, Planellas LL, Caldentey JG, Caballol N, et al. COPPADIS-2015 (COhort of Patients with PArkinson's Dlsease in Spain, 2015): an ongoing global Parkinson's disease project about disease progression with more than 1000 subjects included. Results from the baseline evaluation. Eur J Neurol. 2019;26:1399-407. https://doi.org/10.1111/ ene. 14008 .

17. Hobson DE. Asymmetry in parkinsonism, spreading pathogens and the nose. Parkinsonism Relat Disord. 2012;18:1-9. https://doi.org/10.1016/j. parkreldis.2011.06.011.

18. Riederer $P$, Jellinger KA, Kolber $P$, Hipp G, Sian-Hülsmann J, Krüger R, Lateralization in Parkinson disease. Cell Tissue Res. 2018;373:297-312. https://doi.org/10.1007/s00441-018-2832-z

19. Halliday GM, Leverenz JB, Schneider JS, Adler CH. The neurobiological basis of cognitive impairment in Parkinson's disease. Mov Disord. 2014;29(5):63450. https://doi.org/10.1002/mds.25857.

20. Gunzler SA, Riley DE, Chen SG, Tatsuoka CM, Johnson WM, Mieyal JJ, et al. Motor and non-motor features of Parkinson's disease in LRRK2 G2019S carriers versus matched controls. J Neurol Sci. 2018;388:203-7. https://doi. org/10.1016/j.jns.2018.03.025

21. Boesveldt S, Verbaan D, Knol D, Visser M, van Rooden SM, van Hilten JJ, et al. A comparative study of odor identification and odor discrimination deficits in Parkinson's disease. Mov Disord. 2008;23(14):1984-90. https://doi. org/10.1002/mds.22155.

22. Leonhardt B, Tahmasebi R, Jagsch R, Pirker W, Lehrner J. Awareness of olfactory dysfunction in Parkinson's disease. Neuropsychology. 2019;33(5): 633-41. https://doi.org/10.1037/neu0000544.

23. Mantri S, Morley JF, Siderowf AD. The importance of preclinical diagnostics in Parkinson disease. Parkinsonism Relat Disord. 2019;64:20-8. https://doi. org/10.1016/j.parkreldis.2017.09.010.

24. Sengoku R, Matsushima S, Bono K, Sakuta K, Yamazaki M, Miyagawa S, et al. Olfactory function combined with morphology distinguishes Parkinson's disease. Parkinsonism Relat Disord. 2015;21:771-7. https://doi.org/10.1016/j. parkreldis.2015.05.001.

25. Park JW, Kwon DY, Choi JH, Park MH, Yoon HK. Olfactory dysfunctions in drug-naïve Parkinson's disease with mild cognitive impairment. Parkinsonism Relat Disord. 2018;46:69-73. https://doi.org/10.1016/j.parkreldis. 2017.11.334.

26. Hwang EJ, Ryu DW, Lee JE, Park SH, Choi HS, Kim JS. Magnetic resonance imaging assessment of the substrate for hyposmia in patients with Parkinson's disease. Clin Radiol. 2019; 74(6): 489.e9-489.15. doi: 10.1016/j. crad.2019.02.003.

\section{Publisher's Note}

Springer Nature remains neutral with regard to jurisdictional claims in published maps and institutional affiliations.

\section{Submit your manuscript to a SpringerOpen ${ }^{\circ}$ journal and benefit from:}

- Convenient online submission

- Rigorous peer review

- Open access: articles freely available online

High visibility within the field

- Retaining the copyright to your article

Submit your next manuscript at $\boldsymbol{\text { springeropen.com }}$ 